\title{
Surface States in a Square Lattice under Magnetic Field Applied in the Surface Region
}

\author{
J.W. KŁOS \\ Surface Physics Division, Faculty of Physics, Adam Mickiewicz University \\ Umultowska 85, 61-614 Poznań, Poland
}

\begin{abstract}
Electronic surface states localized on the edge of a semi-infinite square lattice are studied in the tight binding approximation. We examined the existence of surface states in the presence of magnetic field applied in the surface region of a 2D lattice. The applied field is perpendicular to the lattice and confined to a stripe near the surface. We also included a surface site perturbation caused by the presence of the surface. The magnetic field is introduced into the model by the Peierls substitution.
\end{abstract}

PACS numbers: 73.20.-r, 75.70.Rf

\section{Introduction}

The presence of a magnetic field has a significant effect on the spectrum of electronic states in crystal structures. Though long-known $[1,2]$ and quite simple in its formulation [3], this problem remains the subject of intensive research, especially in the field of quantum Hall effect $[4,5]$. Recently, special attention has been paid to systems in which the carrier motion is limited by the surface. Surface effects in quantum dots and quantum wires under magnetic field are of key importance for understanding the transport processes in these systems [6-8].

Based on the tight-binding approximation, the present study is focused on electronic surface states in a semi-infinite square lattice in homogeneous magnetic field confined to a surface stripe of width equal to twice the lattice constant. This model can roughly represent a system with magnetic atoms on the surface. The surface atoms are also assumed to be characterized by a different value of the Coulomb integral, due to dangling bonds and surface reconstruction.

One of the simplest models is a 2D square lattice in the single-band tight-binding approximation. With a suitably chosen vector potential the effect of applied magnetic field is equivalent to modifying resonance by an oscillatory factor $\exp \left(\mathrm{i} m 2 \pi \phi / \phi_{0}\right)$ as a result of translation in the direction tangent to the surface.

This paper presents surface state energy levels calculated versus surface and bulk parameters. The surface parameters include the perturbation of surface atoms and the applied magnetic field (confined to a surface stripe); the bulk parameter considered is the wave-vector component tangent to the surface.

\section{Method}

The wave function of electrons in a square lattice can be expressed in the basis of atomic functions localized at lattice sites $(m, n)$ :

$$
|x, y\rangle=\sum_{m, n} c_{m} d_{n}\left|x_{m}\right\rangle\left|y_{n}\right\rangle,
$$

where $\left|x_{m}\right\rangle=|x-a m\rangle$ and $\left|x_{n}\right\rangle=|x-a n\rangle$. The atomic function $|x, y\rangle$ in (1) is separated into an $x$-dependent part $|x\rangle$ and a $y$-dependent part $|y\rangle$. Left multiplication of the Schrödinger equation by the atomic function $\left\langle x_{m}, y_{n}\right|$ and integration over the whole space leads to the secular equations for coefficients $c_{m}$ and $d_{n}$ [9], which are based on the assumption that the only nonzero elements of the Hamiltonian matrix in the atomic function basis are

$$
\begin{aligned}
& \left\langle x_{-1}, y_{n}|H| x_{-1}, y_{n}\right\rangle=\alpha_{s}, \\
& \left\langle x_{m}, y_{n}|H| x_{m}, y_{n}\right\rangle=\alpha, \quad m>-2, \\
& \left\langle x_{m+1}, y_{n}|H| x_{m}, y_{n}\right\rangle \\
& \quad=\left\langle x_{m}, y_{n}|H| x_{m+1}, y_{n}\right\rangle=-\beta, \quad m>-2, \\
& \left\langle x_{m}, y_{n}|H| x_{m}, y_{n \pm 1}\right\rangle=-\beta \mathrm{e}^{-\mathrm{i} m q}, \quad-2<m<2, \\
& \left\langle x_{m}, y_{n}|H| x_{m}, y_{n \pm 1}\right\rangle=-\beta, m \geq 2 .
\end{aligned}
$$

The coefficients $\alpha, \alpha_{s}$ and $\beta$ are coordinate-independent and refer, respectively, to the Coulomb integral in the bulk, on the surface and the resonance integral for the system in the absence of magnetic field. The model assumes that the magnetic field is perpendicular to the lattice plane and confined to a stripe near the surface $(m=-1,0,1)$. The factor $\exp (-\mathrm{i} m q)$ (in Eq. (2)) results from Peierl's substitution [10]: $k \rightarrow(p+e A) / h$, in the presence of magnetic field where the vector potential has the form: $\boldsymbol{A}=\left[0, A_{y}, 0\right]$. The parameter $q$ describes the magnetic field and is equal to the ratio $\phi / \phi_{0}$ of the magnetic flux per unit cell to the magnetic flux quantum.

As the elements of the Hamiltonian matrix only depend on $x_{m}=m a$, Bloch's theorem can be used for determin- 
ing the relation between coefficients $d_{n}$ :

$$
\frac{d_{n+1}}{d_{n}}=\mathrm{e}^{\mathrm{i} \theta_{y}},
$$

where $\theta_{y}=k_{y} a$ is the (dimensionless) wave vector component tangent to the surface. Using (3), we obtain the following system of equations for coefficients $c_{m}[3]$ :

$$
\begin{cases}c_{0}+2 \cos \left(q+\theta_{y}\right) c_{-1}=-(X+Z) c_{-1}, & m=-1, \\ c_{-1}+c_{1}+2 \cos \left(\theta_{y}\right) c_{0}=-X c_{0}, & m=0, \\ c_{0}+c_{2}+2 \cos \left(q-\theta_{y}\right) c_{1}=-X c_{1}, & m=1, \\ c_{m-1}+c_{m+1}+2 \cos \left(\theta_{y}\right) c_{m}=-X c_{m}, & m \geq 2,\end{cases}
$$

where $X=(E-\alpha) / \beta$ and $Z=\left(\alpha_{s}-\alpha\right) / \beta$ denote the reduced electron energy and the surface perturbation parameter, respectively. With a matrix defined by

$$
\left(\begin{array}{c}
c_{m+1} \\
c_{m}
\end{array}\right)=M^{(m)}\left(\begin{array}{c}
c_{m} \\
c_{m-1}
\end{array}\right),
$$

a coefficient vector inside the lattice, where the magnetic field is zero, can be determined from the boundary conditions included in Eqs. (4):

$$
\left(\begin{array}{c}
c_{2} \\
c_{1}
\end{array}\right)=\underbrace{M^{(1)} M^{(0)} M^{(-1)}}_{M}\left(\begin{array}{c}
c_{-1} \\
0
\end{array}\right) .
$$

Equation (6) allows to find the ratio $c_{2} / c_{1}=M_{1,1} / M_{2,1}$. The magnetic field inside the crystal is zero. Therefore, according to Bloch's theorem, the coefficient ratio $c_{2} / c_{1}$ can be expressed as follows:

$$
\frac{c_{2}}{c_{1}}=\mathrm{e}^{\mathrm{i} \theta_{x}}=\frac{M_{1,1}}{M_{2,1}} .
$$

In the case of surface states the $x$ component of the wave vector $\theta_{x}$ is complex and takes on value [9]:

$$
\theta_{x}=\left\{\begin{array}{r}
\mathrm{i} \mu, \\
\pi+\mathrm{i} \mu,
\end{array}\right.
$$

where $\mu$ is a positive real number.

Surface state energy levels can be found by using dispersion relation: $X=-2 \beta\left[\cos \left(\theta_{y}\right) \pm \cosh (\mu)\right]$ and Eq. (7). Among the results obtained, those which do not imply wave function fading with growing distance from the surface should be eliminated as non-physical. Therefore, according to Bloch's theorem (7), for physical solutions: $\mu>0$.

The dispersion relation implies the existence of surface states of energy $X>-2 \beta\left(1+\cos \theta_{y}\right)$ or $X<2 \beta(1-$ $\left.\cos \theta_{y}\right)$. Assuming $\mu>0$ and considering (7), one can expect the wave function to decrease and change sign at each translation by the lattice constant in the case of surface states above the band, and not to change sign in the case of states below the band.

\section{Results}

Surface state energy $X$ was calculated from dispersion relation and (7) versus the surface perturbation parameter $Z$ at fixed values of magnetic field, described by parameter $q$, and wave vector tangent component $\theta_{y}$ (Fig. 1). The energy range $2 \beta\left(1-\cos \theta_{y}\right)<X<$ $-2 \beta\left(1+\cos \theta_{y}\right)$ represents the band, or energy levels allowed for electrons propagating in the bulk. Forbidden to surface states, this energy range is shaded gray in Fig. 1. The band is seen to shift along the energy axis in an oscillatory manner as the tangent component of the wave vector changes. The calculations were performed at fixed band width, for $\beta=1$. For this parameter value the bottom and the top of the band fall at $\theta_{y}=0$ and $\theta_{y}=\pi$, respectively.

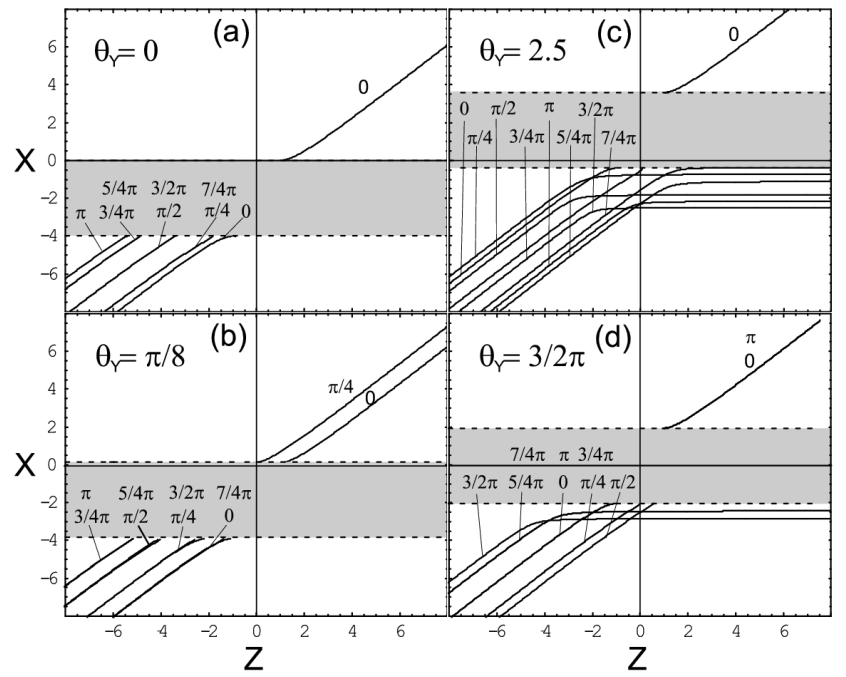

Fig. 1. Spectrum of surface states versus surface perturbation $Z$ for fixed wave vector tangent component $\theta_{y}$ and magnetic field $(q)$. Gray area represents the energy band. Surface state energy levels are labelled with their respective values of $q$. In (a), (b) and (d) degenerate energy levels are seen to occur, each corresponding to two different $q$ values.

Let us first discuss the properties of surface states below the band $\left(X<-2\left(1+\cos \theta_{y}\right)\right)$. For $\theta_{y}$ values close to zero all surface states of energy below the band are induced by negative perturbation $Z$. (The negative value of $Z$ implies that the Coulomb integral for a surface atom is lower than that for a bulk one: $\alpha_{s}<\alpha$ ). Increasing the surface perturbation causes the surface state energy levels to move away from the band edge, with consequent increase in localization of these surface states. The closer the tangent component $\theta_{y}$ of the wave vector to $\pi$ is, the more surface states appear also for $Z>0$. However, their energy (and localization) shows only a slight dependence on the perturbation $Z$. It is worthy of notice that for positive values of $Z$ surface states below the band only exist in the presence of magnetic field $(q \neq 0)$. For $Z<0$ the perturbation necessary to induce a surface state of a given energy shows an oscillatory dependence on $\theta_{y}$ and $q$. Visualized in Fig. 2a (showing the perturbation $Z$ necessary to induce a surface state of energy $X=-4$ versus $\theta_{y}$ and $q$ ), these oscillations are also reflected in the surface state energy plots presented in Fig. 1. For $Z<0$ the order of levels corresponding to 
successive values of $q$ changes as $\theta_{y}$ increases. When the ratio $\left(\theta_{y} \bmod 2 \pi\right) /(q \bmod 2 \pi)$ can be expressed as $j /(2 l)$, where $j$ and $l$ are integers, some of the surface state levels below the band have the same energy (see Fig. 1a, b, d). Otherwise, no level overlapping is seen to occur (Fig. 1c).
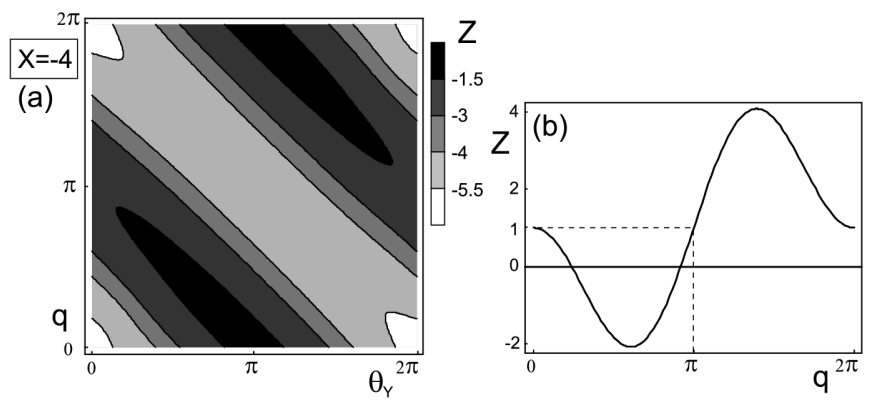

Fig. 2. Critical perturbation $Z$ for surface states with energy (a) below the band and (b) above the band versus wave vector tangent component $\theta_{y}$ and magnetic field $q$. The plots in (a) and (b) refer, respectively, to energy $X=-4$ and $X=2\left(\cos \theta_{y}-1\right)$ of surface state emerging from band. Let us note that in the case of surface states above the band $\theta_{y}$ and $q$ are correlated, fulfilling the condition: $\left(\theta_{y} \bmod 2 \pi\right) /(q \bmod 2 \pi)=1 / 2$.

Surface states above the band $\left(X>2\left(1-\cos \theta_{y}\right)\right)$ only occur when $q=0$ or $\left(\theta_{y} \bmod 2 \pi\right) /(q \bmod 2 \pi)=1 / 2$ (Fig. 1b, d). In the absence of magnetic field $(q=0)$ a surface state is induced by perturbation $Z=1$. If $\left(\theta_{y} \bmod 2 \pi\right) /(q \bmod 2 \pi)=1 / 2$, the perturbation necessary to induce a surface state oscillates with increasing $q$ in a manner shown in Fig. 2b; for $q=\pi$ a surface state emerges from the band at $Z=1$, as can be deduced from the plot. This effect can be seen also in Fig. 1d, where for $\theta_{y}=(3 / 2) \pi$ the level of the surface state occurring in the absence of magnetic field $(q=0)$ corresponds to that of the surface state occurring at magnetic field $q=\pi$. Let us note that the surface state levels move away from the band, with consequent increase in localization, as the perturbation $Z$ increases.

\section{Acknowledgments}

This work was supported by grant N N202194533 from Polish Ministry of Science and Higher Education.

\section{References}

[1] F.A. Butler, E. Brown, Phys. Rev. 166, 630 (1968).

[2] W.G. Chambers, Phys. Rev. 140, A135 (1965).

[3] D.R. Hofstadter, Phys. Rev. B 14, 2239 (1976).

[4] Y. Hasegawa, M. Kohmoto, Phys. Rev. B 74, 155415 (2006).

[5] N. Goldman, P. Gaspard, Phys. Rev. B 77, 024302 (2008).

[6] A. Nikolaeva, D. Gitsu, L. Konopko, M.J. Graf, T.E. Huber, Phys. Rev. B 77, 075332 (2008).

[7] V.Ya. Demikhovskii, D.V. Khomitsky, Phys. Rev. B 67, 035321 (2003).

[8] H.N. Nazareno, P.E. de Brito, E.S. Rodrigues, Phys. Rev. B 76, 125405 (2007).

[9] S.G. Davison, M. Steslicka, Basic Theory of Surface States, Clarendon Press, Oxford 1996, p. 223.

[10] R.E. Peierls, Z. Phys. 80, 763 (1933). 